\title{
Economic Analysis for Selection of Diagnostic Methods against Surra in Buffalo on East Sumba Island, Indonesia
}

\author{
Rita Sari Dewi \\ Indonesian Agricultural \\ Quarantine Agency \\ Ministry of Agriculture \\ Jakarta Indonesia \\ ritawibowo29@gmail.com
}

\author{
April H. Wardhana \\ Department of Parasitology \\ Indonesia Research Center for \\ Veterinary Sciences \\ Bogor, Jakarta \\ wardhana24id@yahoo.com
}

\author{
Retno Damayanti S \\ Department of Animal Disease and \\ Veterinary Public Health \\ Faculty of Veterinary Medicine \\ IPB University \\ Bogor, Indonesia \\ retnodmail@yahoo.com \\ Sri Mulatsih \\ Departement of Economic Science \\ Faculty of Economics and \\ Management \\ IPB University \\ Bogor, Indonesia \\ mulatsupardi@gmail.com
}

\author{
Chaerul Basri \\ Department of Animal Disease and \\ Veterinary Public Health \\ Faculty of Veterinary Medicine \\ IPB University \\ Bogor, Indonesia \\ cherulbasri@gmail.com
}

\begin{abstract}
Surra is one of the main disease problems in buffalo farms in East Sumba Island. The control program of this disease requires the support of diagnostic methods that are reliable and cost-effective. This study aimed to select the diagnostic method for surra based on the economic value of each diagnostic method, including parasitology tests (WBF), Micro-Hematocrit Centrifugation Technique (MHCT), Card Agglutination Trypanosome Test/T. evansi (CATT), and Polymerase Chain Reaction (PCR). Data was collected from various sources including from survey of $\mathbf{3 0}$ buffalo farmers on East Sumba Island, Surra cases from the East Sumba Livestock Service Office, diagnostic test data from the Veterinary Research Institute, Indonesian Ministry of Agriculture and various supporting literatures. The economic value of each diagnostic test was analyzed by the decision tree analysis model using WinQSB software. The results showed that the PCR method has the highest economic value of IDR781.3 million, and then followed sequentially by MHCT IDR.668.4 million, CATT / T. evansi IDR 505.5 million and WBF IDR 114.5 million. Based on this study it is recommended to use PCR as a diagnostic test method in controlling Surra disease in buffaloes on East Sumba by considering its economic value.
\end{abstract}

Keywords- economic, surra, decision tree analysis, diagnostic

\section{INTRODUCTION}

Surra is a disease in animals caused by Trypanosoma evansi (T. evansi). The diseases attack livestock and wild animals such as cows, buffaloes, camels, horses, pigs, dogs, deers, and elephants. The disease is transmitted through the bite of blood-sucking flies (Tabanus sp., Stomoxys sp.), wound contamination, contamination of medical/surgery equipment, sexual activities, and transplacental [1]. Surra occurs in several countries in Asia. Seroprevalence of T. evansi in India reaches $19.6 \%$
[2], and $8.2 \%$ on dairy cattle in Thailand [3]. Cases of Surra in Philippines have increased dramatically causes highly deaths in horses, buffaloes, and cows. Surra outbreaks in Indonesia occurs sporadically, especially in horses, buffaloes, cattle, pigs, and other animals. The pathogenicity of the $T$. evansi from Asian strain is higher than African and American strains, especially in cattle [1].

Control of Surra is a main concern for the world in the context of protecting livestock production. The successful of Surra control program depends on the accurate detection and treatment methods of infected animal [1][4]. The method of diagnostic Surra is important aspects in Surra control program. There are several diagnostic test methods for Surra with their respective advantages and disadvantages. In general, identification of Surra was using parasitology tests (microscopic identification). This technique is simple and inexpensive, but the sensitivity and specificity were very low. This technique only effective in the acute phase when the level of parasitemia was high. Errors in detecting cases can delay treatment and has an impact on increasing morbidity and mortality in the animal population. Other Surra detection methods that are often used are Micro-Hematocrit Centrifugation (MHCT), serological tests and biomolecular techniques. These tests have a higher sensitivity than the parasitology test.

Microscopic identification using the MHCT technique can significantly improve test sensitivity. The serological method that is widely used is Card Agglutination Trypanosome Test /T. evansi (CATT/T. evansi) because of this method is quick and easy. Molecular methods using Polymerase Chain Reaction (PCR) are also developed for detection and confirmation of cases. This technique has been used by several countries in overcoming problems of conventional and serological techniques. Surra diagnosis 
requires at least two types of diagnostic methods to confirm the presence of T. evansi [5] [6]. Each of these tests has a different level of sensitivity. Test methods with a low level of sensitivity will cause a low ability to detect cases of infection. In populations, animals that are infected sub-clinically can be a source of transmission (reservoir) if they cannot be detected. Accurate diagnostic methods in the control program can help to stop the spread of Surra cases in the population.

Economic aspects can be used as one of the considerations in selecting diagnostic methods other than technical aspects. Economic aspect evaluation of laboratory testing has been widely carried out such as the use of PCR in the diagnosis of pulmonary tuberculosis [7] and for molecular diagnostic testing of tuberculosis resistance and rifampicin in Mycobacterium tuberculosis [8]. This study aimed to calculate the economic value of various surra diagnostic methods currently in use, including WBF, MHCT, CATT, and PCR to provide recommendations for the most effective and economical diagnostic methods in the field.

\section{MATERIALS AND METHODS}

\section{A. Data collection}

The study used primary and secondary data. The primary data was about production decline due to Surra and the prices of livestock aspect. The data collected by semi-structured interviews using questionnaires. A total 30 farmer respondents selected from 5 subdistricts out of 19 sub-districts with Surra cases by stratified random sampling. From each subdistrict was chosen $2-3$ villages, and 2-4 farmers were selected from each village. Determination of samples was based on data from the East Sumba Livestock services.

Secondary data collected includes data on costs of the test and prices of laboratory equipment, costs of operator for training and maintenance of the equipments. Data obtained from the Bogor Veterinary Research Center and providers of laboratory equipments.

\section{B. Economic Evaluation}

The economic evaluation was calculated using the level of sensitivity of each diagnostic method. The sensitivity of a test is the proportion of animals that are really infected in the population who also give positive results based on the test. The level of sensitivity is used as a probability value to give positive results to animals that are actually infected. Thus a high level of sensitivity will cause infected animals (clinical and subclinical) which can be detected will increase. The probability of an infected animal not being detected by a diagnostic method (false negative) is $100 \%$ minus the level of test sensitivity.

The false negative probability is the possibility of the number of animals infected but not detected (sub-clinical) and not treated so that it has the potential to spread the disease in its population. The probability of subclinical cases will die about $17.5 \%$ and as much as $50 \%$ will turn into clinical cases [9]. The probability of a clinical case turn to death is $25 \%$ and the remaining $75 \%$ is assumed to have a decrease in production (body weight). Based on this description an economic analysis framework was developed, as shown in Fig. 1.

Economic losses are obtained by multiplying the probability with the price of buffalo IDR 5,900,000. To test the sensitivity of the results of economic analysis, a sensitivity analysis was carried out in this study using maximum and minimum buffalo price parameters (IDR $5,400,000-6,400,000)$

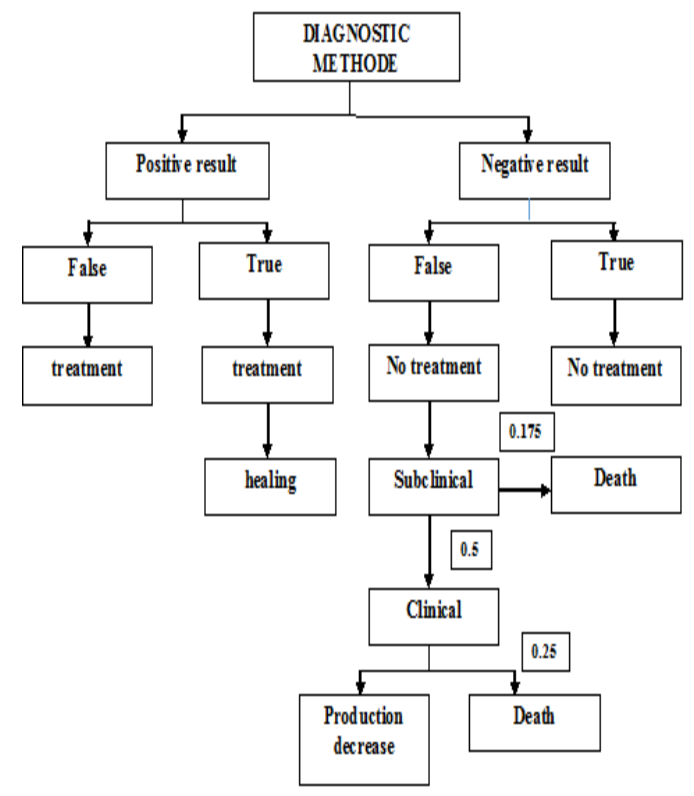

*probability base on Dubson et
Fig. 1. Framework economic analysis of Diagnostic method of Surra

An economic evaluation of 4 types of Surra diagnostic methods that are often used in the field are WBF, MHCT, CATT/T. evansi, and PCR. Decision tree analysis was chosen as an evaluation method [7] [10]. Decision tree analysis can be used if there are several possible outcomes from several choices of actions, and probabilities are important factors to determine outcomes. The tree analysis method will give the best results with the highest economic value or called expected economic value (EMV). Economic calculations use the assumption of the number of samples 1000 per year. The prevalence used for Surra was $15.3 \%$ [11]. The probability is obtained from the sensitivity values of each test based on the references as in Table 1 .

Laboratory costs of each diagnostic method are calculated and compared. This costs consist of the cost of testing per sample, the cost of purchasing laboratory materials, the investment costs for PCR tools and microscopes, and the maintenance costs for the equipment per year. For laboratory methods that require special skills, operator training costs are added. Investment costs are calculated by the cost of the purchase divided by the time the device is used (10 years) (Table 1 ).

The decision tree analysis model shows the pathway for each possible outcome obtained from each diagnostic method. Each pathway is equipped with a probability value and a resulting economic value. The results of this analysis model can be used to decide the best diagnostic method based on the highest economic value. The decision tree 
analysis model was created using WinQSB software, with the input as shown in Tables 1 and 2.

TABLE I PARAMETER FOR DECISION TREE ANALYSIS

\begin{tabular}{|c|c|c|c|c|c|}
\hline Parameter & & PCR & CATT & MHCT & WBF \\
\hline $\begin{array}{l}\text { Price of water } \\
\text { buffaloes/animal (Rp) }\end{array}$ & $9000000^{1}$ & & & & \\
\hline Sample number & 1000 & & & & \\
\hline Prevalence Surra & $15.3 \%^{2}$ & & & & \\
\hline Estimated positive result & 153 & & & & \\
\hline $\begin{array}{l}\text { Test probability }(\%) \\
\text { (sensitivity) } \\
\text { Laboratory cost }(000)^{7}\end{array}$ & & $93.8^{3}$ & $60^{4}$ & $69.6^{5}$ & $13.8^{6}$ \\
\hline - $\quad 1000$ sample (Rp) & & 412196 & 16000 & 5000 & 5000 \\
\hline $\begin{array}{l}\text { Laboratory equipment } \\
\text { depreciation per year }\end{array}$ & & 26940 & & 5000 & 5000 \\
\hline - Maintenance & & 10000 & & 500 & 500 \\
\hline - Operator training & & 10000 & & & \\
\hline
\end{tabular}

TABLE II. INPUT FOR DECISION TREE ANALYSIS

\begin{tabular}{llcccc}
\hline \multirow{2}{*}{ Criteria } & \multicolumn{1}{c}{ Formula } & \multicolumn{4}{c}{ Economic value (000) } \\
\cline { 3 - 6 } & & PCR(1) & CATT(2) & MHCT(3) & WBF(4) \\
\hline Positive & (Pn x J x H)-Cn & 832489 & 810200 & 962418 & 179526 \\
False-negative & $(1-P n) \times$ J & - & - & - & - \\
$\begin{array}{l}\text { Death false negative } \\
\text { Clinic false negative }\end{array}$ & $0.175 \times$ AnNP x H & 14175 & 96075 & 72450 & 207900 \\
$\begin{array}{l}\text { Subclinical false } \\
\text { negative }\end{array}$ & 0.325 x nNP & - & - & - & - \\
$\begin{array}{l}\text { Death clinics false } \\
\text { negative }\end{array}$ & $0.25 \times$ AnNPK x H & 11250 & 69750 & 51750 & 148500 \\
$\begin{array}{l}\text { Production decline } \\
\text { clinics false negative }\end{array}$ & $0.75 \times$ AnNPK x PP & 9923 & 61519 & 45643 & 130977 \\
\hline
\end{tabular}

\section{RESULTS}

Since the first Surra outbreak at the end of 2010, East Sumba has not been back to free from this disease. The cases still sporadically occurs especially on horses and buffaloes. The control program needs to be evaluated to overcome this situation. Improving of the diagnostic method which more sensitive could be one step that can be applied.

In addition to the technical aspects, economic aspects can be one of the considerations in determining of the diagnostic method against Surra. With simple and inexpensive considerations, so far the parasitology test is still used to detect surra even though the sensitivity and specificity are low. In this study, the economic aspect is used as a consideration to determine the Surra diagnostic method. The economic value calculation of each method based on technical aspects, especially the value of sensitivity (Table 2).

Decision making to choose the diagnostic method based on economic evaluation is done using a decision tree analysis model as shown in Fig. 2. Nodes 2,3 and 4 shown several alternative choices of diagnostic methods for surra with their economic value seen at the bottom of each of the nodule (red color). The percentage value as shown on each line is the probability as explained in Table 1 . The best choice are found in red nodules with the highest economic value.

The highest cost-effectiveness is in the PCR method, the expected monetary value is IDR 781.3 million followed by
MHCT IDR 668.4 million. The lowest results on the WBF method, Rp. 114.5 million, while CATT/T. evansi gives an economic value of Rp.668.5 million. In the WBF method which has the lowest sensitivity, only $13.8 \%$ can detect sick animals (positive test) so that the economic value is very low. In the sensitivity test using the highest and lowest buffalo price parameters, giving the same results as the highest EMV sequence is PCR, MHCT, CATT/T. evansi and WBF (Table 3).

\begin{tabular}{lcc} 
& TABLE III. TABLE DATA SENSITIVITY \\
\hline Test methods & High price (IDR) & Low price (IDR) \\
\hline PCR & 565877100 & 942948800 \\
CATT & 413976600 & 574248800 \\
MHCT & 548069100 & 758188300 \\
WBF & 98528600 & 129931800 \\
\hline
\end{tabular}

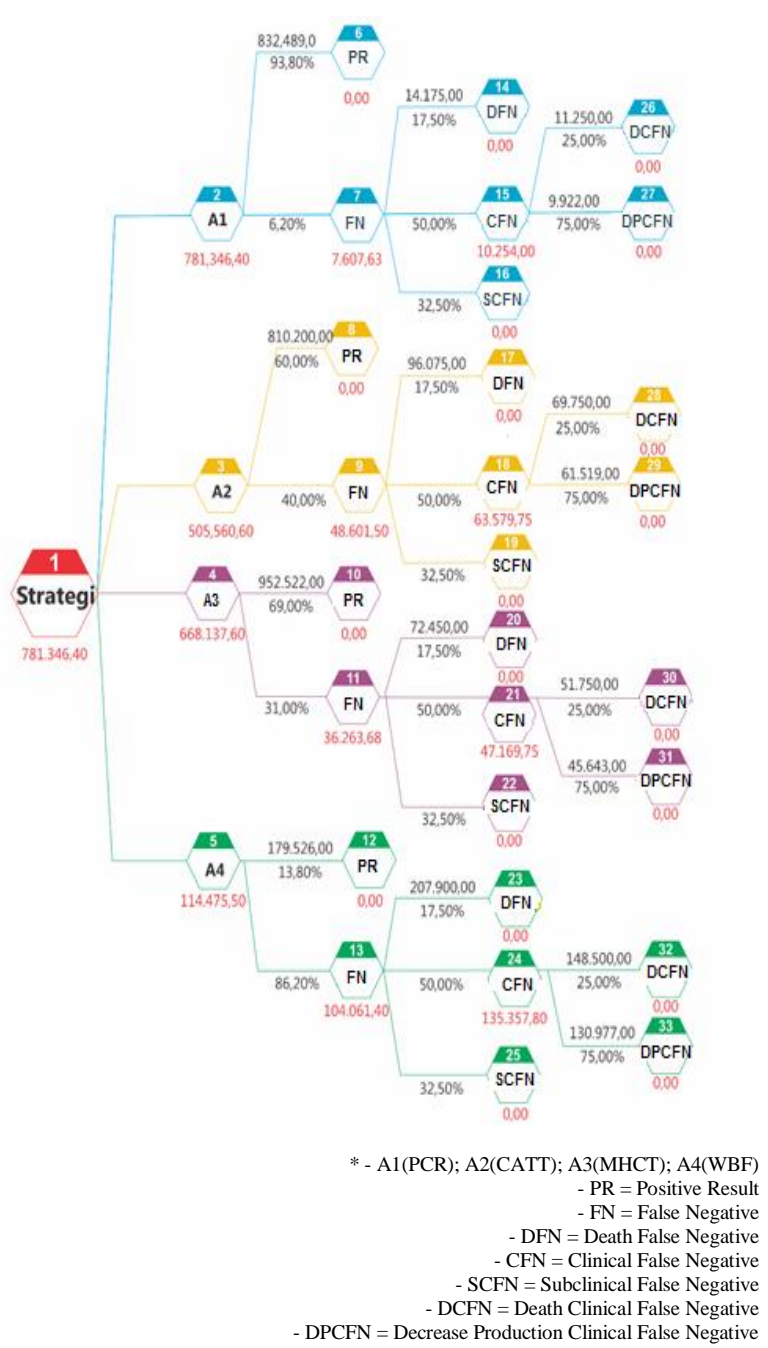

Fig. 2. Decision tree analysis for diagnostic methods of Surra

\section{DISCUSSION}

Detection of the presence of Trypanosoma in the blood has become the gold standard, but finding organisms in the blood is not easy, even in state of parasitemia and shows of clinical symptoms [13]. The benefits of a blood test are simple and inexpensive, and if Trypanosoma is found then the disease can be diagnosed on the spot. The disadvantage of this diagnostic method is that swab samples taken to 
veterinary laboratories will lose their mobility over a period of time and their sensitivity level will be limited to around $10^{4}$ Trypanosomas per $\mathrm{ml}$ of blood. The diagnostic test standard for T. evansi detection in Indonesia is a thin blood swab with the sensitivity of $10^{5}$ Trypanosoma $/ \mathrm{ml}$ of blood. Therefore the sensitivity of parasite detection to be increased for example by using HCT with the sensitivity of 85 Trypanosoma/ml [14]. The accuracy of the method is only $13.8 \%$, which causes many positive animals undetected and untreated. They are potential as a reservoir or source of transmission in group of animals.

The MHCT method usually use as the first test because this method is highly sensitive. If the result of MHCT shows negative then the test is continued with PCR method [15]. Serologic methods usually give better results than parasitological method. Technically, the CATT/T. evansi method has the advantage over parasitology method because it has a higher sensitivity value of around $60 \%$. In addition, CATT / T. evansi is easy to carry out in the field and easy to apply even though reading the results requires experience.

Another obstacle for this method is the availability of CATT/T. evansi is still very limited. Evaluation of serological tests against Surra on buffaloes in Indonesia showed CATT /T. evansi gives the best results [16]. WHO also recommends the use of serological tests (micro-card agglutination test for Trypanosomosis) [17]. CATT/T. evansi detects Surra through the presence of immunoglobulin-M in the blood so that it can be used to detect in the early phase of infection. Whereas, ELISA is usually used for the detection of immunoglobulin-G [18]. In choosing a test method, consideration should be given to the cost effectiveness and time availability because there is no serological testing method that gives $100 \%$ sensitivity [15].

Biomolecular methods have been widely used in detecting T. evansi, as it is known that PCR has a $93.8 \%$ sensitivity test [19][6][20]. With this method, in minutes DNA of the parasite will amplify so that the amount is sufficient to be detected depending on the primer used. Some primers can be used specifically for sub-species, types and even strains. Comparative studies recommend TBR primer as the most sensitive primer for the detection of T. evansi [21] and the phenol-chloroform method is the most sensitive method for DNA isolation. This method can detect at least 5-10 Trypanosomas per $\mathrm{ml}$ of blood or with low infection rate [21] [22].

Molecular techniques such as PCR, in the case of latent infections, give promising results with a higher level of sensitivity and specificity and are very good when used for epidemiological studies of T. evansi infections [22]. This biomolecular technique has developed and is widely used in several countries to overcome the limitations of conventional and serological tests. But in diagnosis, Surra requires at least two diagnostic tests to confirm the presence of $T$. evansi in the infected animals [5][6]. In determining of the status of Surra-free areas, the CATT serial test is recommended, and or followed by retest the sampel, preferably when equipped with PCR [23].
Since the first Surra outbreak in 2010, East Sumba has not been free from Surra, the East Sumba government must make some improvements in the Surra control including the diagnostic methods used. Although there are several alternative diagnostic methods for Surra, it is recommended to use the PCR method in addition to the parasitological method in the survey and control program, especially for diagnosing cases [24]. The most sensitive method for giving the highest economic value is PCR. The diagnostic method that requires high investment costs turns out to be the most economical diagnostic method because it provides more benefits (cost-effectiveness) so it is feasible to use.

Based on the assumption of 1000 samples taken and the prevalence of Surra in East Sumba 15.3\% (153 positive samples). Each test method can detect case positive Surra according to the sensitivity level of each method. If the sensitivity is $100 \%$, the test method is expected to detect 153 positive cases. PCR with a sensitivity value of $93.8 \%$ will only be able to detect $93.8 \%$ of 153 infected animals, while the rest are false negatives. As shown in Figure 1, undetected infected animals (false negatives) have the potential to become clinical cases or die.

The number of undetectable sick animals is 66 animals (WBF), 24 animals (MHCT), 31 animals (CATT/T. evansi) and PCR 4 animals. These values indicate the number of animals that can be reservoirs that spread disease. The higher of the reservoirs number, the higher of the Surra transmission risk and the greater the potential economic losses caused.

Based onthe study that one infected animal in a group of 100 healthy animals could spread infection throughout the group within 12 weeks with 50 insects/ animals/day. If we use the experiment as an illustration of disease transmission, the highest loss occurs when using the WBF test, 66 undetected animals will cause 6600 livestock to be infected with Surra within 12 weeks, while the smallest loss using PCR is only 6 animals that have the potential to infect Surra [25].

The use of appropriate testing methods can reduce the number of sick animals by providing treatment to animals that are sick and can reduce the number of reservoirs in groups of livestock. The use of testing methods that require high investment and expensive materials is more economical because it reduces losses due to disease.

\section{CONCLUSION}

The economic evaluation using the decision tree model showed PCR method had the highest expected economic value (EMV) in total Rp781.3 million followed by MHCT with total Rp.668.4 million and CATT / T. evansi with total Rp. 505.5 million. The WBF has the lowest EMV with a total of Rp.114.5 million. PCR is recommended to be the main diagnostic method for the Surra case based on economic value.

\section{ACKNOWLEDGMENT}

This work is part of the Ph.D. Dissertation by the first author and we are grateful to the farmers, The Livestock 
Services of Sumba Timur and The Disease Investigation Center of Denpasar for their help in providing the data for this study, and the Indonesia Agricultural Quarantine Agency-The Ministry of Agricultural for its financial support.

\section{REFERENCES}

[1] M. Desquesnes, A. Dargantes, D. Lai ,Z.R. Lun, P. Holzmuller, S. Jittapalapong. "Trypanosoma evansi and Surra: a review and perspectives on transmission, epidemiology and control, impact, and zoonotic aspects", BioMed Res Int, 2013, pp. 1-20.

[2] R. Kumar, S. Kumar, S. Khurana, S. C. Yadav. "Development of an antibody-ELISA for seroprevalence of Trypanosoma evansi in equids of North and North-Western regions of India". Veterinary Parasitology, 2013, Vol. 196(3-4), pp. 251-257.

[3] M. Desquesnes, F. Biteau-Coroller, J. Bouyer, M. L.Dia, L. Foil. "Development of a mathematical model for mechanical transmission of trypanosomes and other pathogens of cattle transmitted by tabanids", Int. J. for Parasitol, 2009, Vol. 39, pp. 333-346.

[4] T. T. Nguyen, M. Zhou, N. Ruttayaporn, Q. D. Nguyen, V. K Nguyen, Y. Goto, Y. Suzuki, Si. Kawazu, N. Inoue. "Diagnostic value of the recombinant repeat antigen TeGM6-4r for Surra in water buffaloes". Vet. Par, 2014, Vol. 201, pp. 1-2.

[5] J. L. Gonzales, E. Chacon, M. Miranda, A. Loza, L. M. Siles. "Bovine trypanosomosis in the Bolivian Pantanal. Vet Parasitol, 2007, Vol. 146, pp. 9-16.

[6] El. Metanaway, T. M. Nadia, M. El-Beih, E. A. Abdel, M. S. Hassanane, T. H Aziz, "Comparative studies on Diagnosis of Trypanosoma evansi Experimentally Infected goats". Global Vet. 2009, Vol. 3(4), pp. 348-353.

[7] I. Rajalahti, E L. Ruokonen, T. Kotomaki, H. Sintonen, M. M Nieminen. "Economic evaluation of the use of PCR assay in diagnosing pulmonary TB in a low incidence area", Eur Respir $J$. 2004, Vol. 23, pp. 446-451.

[8] F. A. Drobniewski, S. A. Watterson, S. M. Wilson, G. S Harris. "A clinical, microbiological and economic analysis of a national service for rapid molecular diagnosis of tuberculosis and rifampicin resistance in Mycobacterium tuberculosis". J. Med. Microbiol,

[9] 2000, Vol. 49, pp. 271-278.

[10] R. J. Dobson, A. P. Dargantes, R. T. Mercado, S. A. Reid, Models for Trypanosoma evansi (Surra), its control and economic impact on small-hold livestock owners in the Philippines, 2009, Vol. 39, pp. 1115-1123.

[11] R. B. M. Huirne, A. A. A. "Dijkhuizen. Basic methods of economic analysis animal health economics". Post Graduate Foundation in Veterinary Science. Sydney (AU), 1997.

[12] I.K. Mastra IK. 2011. Seroprevalensi Trypanosomiasis di Pulau Sumba wa. Propinsi Nusa Tenggara Barat. Bul Vet BBVet Denpasar XXIII, 131:138

[13] W. G. Holland, F. Claes, L. N. My, N. G. Thanh, P. T. Tam, D. Verloo, P. Buscher, B. Goddeeris, J. Vercyursse. "A comparative evaluation of parasitological test and a PCR for Trypanosoma evansi diagnosis in experimentally infected water buffaloes". Vet Parasitol. 2001, Vol. 97, pp. 23-33.

[14] M. A. Muieed, Z.I. Chaudhary, A. R. Shakoori. "Comparative studies on the sensitivity of polymerase chain reaction (PCR) and microscopic examination for the detection of Trypanosoma evansi in horses", Turk J Vet Anim Sci, 2010, Vol. 34(6), pp. 507-512.

[15] S. A. Reid SA, "Trypanosoma evansi control and containment in Australia", Review, Trends Parasitol, 2002, Vol. 18(5), pp. 209224

[16] M. T. Tejedor-Junco, M. Gonzales, N. F. Rodriguez, J. A. Corbera, C. Gutierrez, "Polymerase chain reactin (PCR) to detect T. evansi in experimentally inoculated goats", Small Ruminant Res, 2011, Vol. 96, pp: 70-72.

[17] H. C. Davison, M. Thrusfield, S. Muharsini, A. Husein, S. Partoutomo, P. F. Rae, R. Masake, A. G. Luckins, "Evaluation of antigen detection and antibody detection tests for Trypanosoma evansi infections of buffaloes in Indonesia", Epid Infect, 1999, Vol. 123 (1), pp. 149-155.

[18] P. Truc, V. Lejon, E. Magnus, V. Jammonneau, A. Nangouma, Verloo, L. Penchenier, P. Buscher, "Evaluation of the micro-CATT, CATT/Trypanosoma brucei gambiense, and LATEX/T. $b$. gambiense methods for serodiagnosis and surveillance of human African trypanosomiasis in West and Cetral Africa", Bull world Health Organization, 2002, Vol. 80(11), pp. 882-886.

[19] A. K. Jaiswal, V. Sudan, Neha, K. Verma K, Insight into trypanosomiasis in animals: Various approaches for its diagnosis, treatment and control: a review. Asian J Anim Sci. 2015, Vol. 9(5), pp. $172-186$.

[20] F. Claes, M. Radwanskw, T. Urakawa, P. A. O. Majiwa, B Goddeeris, P. Buscher, 2004, "Variable surface glycoprotein Ro Tat $1.2 \mathrm{PCR}$ as a specific diagnostic tool for detection of $T$. evansi infection", Kinetoplastid Biol Dis, 2004, Vol. 3 (3), pp. 1-6.

[21] S. Bashir, M. A. Bakheit, E. Sugimoto, "An overview on the economic impacts of animal trypanosomosis in Sudan", Vet Parasitol, 2014, Vol. 200, pp 246-250.

[22] M. Pruvot, K. Kamyingkird, M. Desquesnes, N. Sarataphan, S. Jittapalapong. "The effect of the DNA preparation method on the sensitivity of PCR for detection of Trypanosoma evansi in rodents and implications for epidemiological surveillance efforts", Vet. Par, 2013, Vol. 191, pp. 203-308.

[23] D. Sumbria, L. D. Singla, A. Sharma, M. S. Bal, Kumar, "Multiplex PCR for detection of Trypanosoma evansi and Theileria equi in equids of Punjab, India", Vet Parasitol, 2015, Vol. 211(3-4), pp. 293-299.

[24] [OIE] Organization Internationale de Epizootic, "Trypanosoma evansi infection: OIE Terrestial Manual Chap.2.1.17. France (FX) "The World Organization for Animal Health, 2012, doi: $10.1080 / 01652176.1986 .9694049$

[25] S. G. Ghattas, N. M. Helmy, "Parasitological and molecular studies on Trypanosoma evansi of camels in Egypt". Egypt J. Agric. Res. 2016. Vol. 94(1), pp. 121-130.

[26] M. Desquesnes, K. Kamyingkird, M. Pruvot, "Antibody-ELISA for Trypanosoma evansi: application in a serological survey of dairy cattle, Thailand, and validation of a locally produced antigen", Preventive Vet Med, 2009, Vol. 90 (3-4), pp. 233-241. 